\title{
A Uniform Distribution Question Related to Numerical Analysis
}

\author{
By Harald Niederreiter* and Charles F. Osgood
}

Abstract. Using the theory of uniform distribution modulo one, it is shown that under certain conditions on the real-valued functions $\alpha(x)$ and $g(x)$ on $[0,1]$,

$$
h \sum_{\gamma=1}^{\left|h^{-1}\right|}\left\{h^{-1} \alpha(\gamma h)\right\}^{m} g(\gamma h)=(m+1)^{-1} \int_{0}^{1} g(x) d x+O\left(h^{1 / 3} \log \frac{1}{h}\right) \quad \text { as } h \rightarrow 0+,
$$

where $m>0$ and $\{x\}$ denotes the fractional part of $x$. The conditions are as follows: $\alpha^{\prime \prime}(x)$ exists for all but finitely many points in $[0,1]$, changes sign at most finitely often, and is bounded away in absolute value from both 0 and $\infty$, whereas $g(x)$ is of bounded variation on $[0,1]$. Also, under these conditions on $\alpha(x)$,

$$
h \sum_{\gamma=1}^{\left|h^{-1}\right|}\left\{h^{-1} \alpha(\gamma h)\right\}^{m}=(m+1)^{-1}+O\left(h^{1 / 3}\right) \quad \text { as } h \rightarrow 0+.
$$

These results, which are, in fact, deduced from somewhat more general propositions, answer questions of Feldstein connected with discretization methods for differential equations.

In connection with algorithms for solving retarded ordinary differential equations by discretization methods, Feldstein [1] was led to search for conditions on the realvalued functions $\alpha(x)$ and $g(x)$ defined on $[0,1]$ which guarantee that

$$
\lim _{h \rightarrow 0+} \int_{0}^{1}\left\{h^{-1} \alpha(x)\right\}^{m} g(x) d x=\frac{1}{m+1} \int_{0}^{1} g(x) d x
$$

for $m>0$, where $\{x\}=x-[x]$ is the fractional part function. Sufficient conditions of a quite general nature were given in [1]. The question was raised as to what happens when the integral on the left-hand side is replaced by a Riemann sum of mesh size $h$. We address ourselves to this problem. Thus, we inquire about conditions on $\alpha(x)$ and $g(x)$ in order for

$$
\lim _{h \rightarrow 0+} h \sum_{\gamma=1}^{\left[h^{-1}\right]}\left\{h^{-1} \alpha(\gamma h)\right\}^{m} g(\gamma h)
$$

to exist and equal $(m+1)^{-1} \int_{0}^{1} g(x) d x$, and furthermore, what could be said about the rate of convergence.

Since it is straightforward to check that for $m>0, g(x) \equiv 1$, and $\alpha(x)=x+a$ with a constant $a \neq 0$, the limit in (1) fails to exist, it is clear that some restrictions on the derivatives of $\alpha(x)$ are to be expected. Actually, the simple condition on the second derivative of $\alpha(x)$ enunciated in Theorem 1 below will suffice (compare with

Received January 14, 1975 ; revised September 24, 1975.

AMS (MOS) subject classifications (1970). Primary $10 \mathrm{~K} 05,65 \mathrm{~L} 05$; Secondary $34 \mathrm{~K} 99$.

* The research of this author was supported in part by NSF grant MPS 72-05055A02. 
Corollary 2). The regularity conditions on $g(x)$ are rather mild, since $g(x)$ need only be of bounded variation on $[0,1]$. If these requirements are met, an estimate for the remainder term can also be established. The condition on $\alpha^{\prime \prime}(x)$ can even be relaxed somewhat (see the remark following Corollary 3).

The reader may check that, using the proofs presented below, the implied constant in the error estimate in Corollary 2 may be effectively computed in terms of the upper and lower bounds on $\left|\alpha^{\prime \prime}(x)\right|$, the total variation of $g(x)$ on $[0,1]$, and $\sup _{0 \leqslant x \leqslant 1}|g(x)|$, and in Corollary 3 in terms of the upper and lower bounds on $\left|\alpha^{\prime \prime}(x)\right|$ alone. It is interesting to note that the constants turn out to be independent of $m$. This is due to the fact that the function $x^{m}$ has total variation 1 on $[0,1]$, no matter which $m>0$ we consider.

The results concefrning (1) are deduced from somewhat more general statements contained in Theorem 1 and, for $g(x) \equiv 1$, in Theorem 2 . In the proofs of these theorems, we need the notions of discrepancy of a finite sequence of points in $[0,1]^{2}$ and in $[0,1]$, respectively, for which we refer to [3, Chapter 2 , Section 1]. We recall also that a function $F(x, y)$ on $[0,1]^{2}$ is said to be of bounded variation in the sense of Hardy and Krause if it is of bounded variation on $[0,1]^{2}$ in the sense of Vitali (see [2] and [3, Definition 5.1, p. 147]), and if the four functions $F(x, 0)$, $F(x, 1), F(0, y)$, and $F(1, y)$ are of bounded variation on $[0,1]$. We shall use Vinogradov's notation $<<$ and Landau symbols interchangeably.

THEOREM 1. Let $F(x, y)$ be of bounded variation on $[0,1]^{2}$ in the sense of Hardy and Krause, and let $\alpha(x)$ be twice differentiable on $[0,1]$ with $A \leqslant \alpha^{\prime \prime}(x) \leqslant B$ for $0 \leqslant x \leqslant 1$ or $-B \leqslant \alpha^{\prime \prime}(x) \leqslant-A$ for $0 \leqslant x \leqslant 1$ and positive constants $A$ and $B$. Then,

$$
h \sum_{\gamma=1}^{\left[h^{-1} l\right.} F\left(\left\{h^{-1} \alpha(\gamma h)\right\}, \gamma h\right)=\int_{0}^{1} \int_{0}^{1} F(x, y) d x d y+O\left(h^{1 / 3} \log \frac{1}{h}\right) \text { as } h \rightarrow 0+.
$$

Proof. Let $h$ be sufficiently small, say $0<h \leqslant 1 / 8$, and let $M=\left[h^{-1 / 3}\right]$. For integers $j$ and $k$ we set

$$
S_{j, k}=\left|\frac{1}{\left[h^{-1}\right]} \sum_{\gamma=1}^{\left|h^{-1}\right|} \exp \left[2 \pi i\left(j h^{-1} \alpha(\gamma h)+k \gamma h\right)\right]\right| .
$$

If $1 \leqslant|j| \leqslant M$, we write

$$
S_{j, k}=\left|\frac{1}{\left[h^{-1}\right]} \sum_{\gamma=1}^{[h-1} \exp [2 \pi i q(\gamma)]\right|
$$

where the function $q$ is defined by $q(t)=j h^{-1} \alpha(h t)+k h t$ for $1 \leqslant t \leqslant\left[h^{-1}\right]$. Of course, $q$ depends also on $h, j$, and $k$, but we suppress this dependence in the notation. We observe that $q^{\prime \prime}(t)=j h \alpha^{\prime \prime}(h t)$, so that $q^{\prime \prime}(t)$ does not change sign and $\left|q^{\prime \prime}(t)\right| \geqslant$ $|j| h A>0$. Therefore, by (3) and an inequality of van der Corput (see [3, Chapter 1, Theorem 2.7] and [5]) we have

$$
\begin{aligned}
S_{j, k} & \leqslant\left[h^{-1}\right]^{-1}\left(\left|q^{\prime}\left(\left[h^{-1}\right]\right)-q^{\prime}(1)\right|+2\right)\left(4(|j| h A)^{-1 / 2}+3\right) \\
& =\left[h^{-1}\right]^{-1}\left(\left|j \alpha^{\prime}\left(h\left[h^{-1}\right]\right)-j \alpha^{\prime}(h)\right|+2\right)\left(4(|j| h A)^{-1 / 2}+3\right) .
\end{aligned}
$$


From $\left|\alpha^{\prime \prime}(x)\right| \leqslant B$ and the mean-value theorem we infer

$$
\left|j \alpha^{\prime}\left(h\left[h^{-1}\right]\right)-j \alpha^{\prime}(h)\right| \leqslant|j| B .
$$

Since $|j| h \leqslant M h \leqslant h^{2 / 3} \leqslant 1 / 4$, we have

$$
4(|j| h A)^{-1 / 2}+3 \leqslant\left(4 A^{-1 / 2}+3 / 2\right)(|j| h)^{-1 / 2}
$$

and so,

$$
S_{j, k}<<(|j| h)^{1 / 2} \text { for } 1 \leqslant|j| \leqslant M \text { and all } k,
$$

where the implied constant is independent of $h, j$, and $k$. For $j=0$ and $1 \leqslant|k| \leqslant M$, we have

$$
S_{0, k}=\left|\frac{1}{\left[h^{-1}\right]} \sum_{\gamma=1}^{\left|h^{-1}\right|} e^{2 \pi i k \gamma h}\right|=\frac{\left|e^{\left.2 \pi i k h \mid h^{-1}\right]}-1\right|}{\left[h^{-1}\right]\left|e^{2 \pi i k h}-1\right|} .
$$

Now $|k| h \leqslant M h \leqslant h^{2 / 3} \leqslant 1 / 4$ and $|k|-|k| h<|k| h\left[h^{-1}\right] \leqslant|k|$, so that for certain $\delta$ and $\theta$ with $0 \leqslant \delta<\theta \leqslant 1 / 4$ we have

$$
S_{0, k}=\left|e^{2 \pi i \delta}-1\right| /\left[h^{-1}\right]\left|e^{2 \pi i \theta}-1\right|
$$

and hence,

$$
S_{0, k}<\left[h^{-1}\right]^{-1}<<h \quad \text { for } 1 \leqslant|k| \leqslant M .
$$

Let $r(a)=\max (1,|a|)$ for $a \in \mathbf{Z}$. By a result on quantitative Fourier inversion (see [4]), the discrepancy $D_{\left[h^{-1}\right]}$ of the points $\left(\left\{h^{-1} \alpha(\gamma h)\right\}, \gamma h\right), \gamma=1,2, \ldots,\left[h^{-1}\right]$, in $[0,1]^{2}$ satisfies

$$
D_{\left|h^{-1}\right|}<<\frac{1}{M}+\sum_{|j| \leqslant M ;|k| \leqslant M}^{*} \frac{1}{r(j) r(k)} S_{j, k}
$$

where the asterisk indicates that $(j, k)=(0,0)$ is deleted from the range of summation and where the implied constant is absolute. It follows now from (4) and (5) that

$$
\begin{aligned}
D_{\left|h^{-1}\right|} & <\frac{1}{M}+\sum_{1 \leqslant|j| \leqslant M ;|k| \leqslant M} \frac{1}{|j| r(k)} S_{j, k}+\sum_{1 \leqslant|k| \leqslant M} \frac{1}{|k|} S_{0, k} \\
& <\frac{1}{M}+h^{1 / 2} \sum_{1 \leqslant|j| \leqslant M}|j|^{-1 / 2} \sum_{|k| \leqslant M} \frac{1}{r(k)}+h \sum_{1 \leqslant|k| \leqslant M} \frac{1}{|k|} \\
& <\frac{1}{M}+h^{1 / 2} M^{1 / 2} \log M+h \log M<<h^{1 / 3}+h^{1 / 3} \log \frac{1}{h}<h^{1 / 3} \log \frac{1}{h},
\end{aligned}
$$

by the definition of $M$. According to [3, Chapter 2, Theorem 5.5], we get

$$
\frac{1}{\left[h^{-1}\right]} \sum_{\gamma=1}^{\left|h^{-1}\right|} F\left(\left\{h^{-1} \alpha(\gamma h)\right\}, \gamma h\right)=\int_{0}^{1} \int_{0}^{1} F(x, y) d x d y+O\left(h^{1 / 3} \log 1 / h\right) .
$$

Since $F$ is bounded, we have

$$
h \sum_{\gamma=1}^{\left[h^{-1}\right]} F\left(\left\{h^{-1} \alpha(\gamma h)\right\}, \gamma h\right)=\frac{1}{\left[h^{-1}\right]} \sum_{\gamma=1}^{\left[h^{-1}\right]} F\left(\left\{h^{-1} \alpha(\gamma h)\right\}, \gamma h\right)+O(h),
$$


and the desired result follows.

Corollary 1. Let $f(x)$ and $g(x)$ be of bounded variation on $[0,1]$, and let $\alpha(x)$ satisfy the conditions of Theorem 1. Then,

$$
\begin{aligned}
& h \sum_{\gamma=1}^{\left|h^{-1}\right|} f\left(\left\{h^{-1} \alpha(\gamma h)\right\}\right) g(\gamma h) \\
& \quad=\left(\int_{0}^{1} f(x) d x\right)\left(\int_{0}^{1} g(x) d x\right)+O\left(h^{1 / 3} \log 1 / h\right) \text { as } h \rightarrow 0+.
\end{aligned}
$$

Proof. It suffices to note that $F(x, y)=f(x) g(y)$ is of bounded variation on $[0,1]^{2}$ in the sense of Hardy and Krause. The rest follows from Theorem 1.

Corollary 2. Let $m>0$ be real, and let $g(x)$ and $\alpha(x)$ be as in Corollary 1 . Then,

$$
h \sum_{\gamma=1}^{\mid h^{-1} l}\left\{h^{-1} \alpha(\gamma h)\right\}^{m} g(\gamma h)=\frac{1}{m+1} \int_{0}^{1} g(x) d x+O\left(h^{1 / 3} \log 1 / h\right) \quad \text { as } h \rightarrow 0+.
$$

Proof. Take $f(x)=x^{m}$ in Corollary 1 .

In the special case where $g(x)$ is constant, say $g(x) \equiv 1$, slight improvements on the above results can be obtained.

THEOREM 2. Let $f(x)$ be of bounded variation on $[0,1]$, and let $\alpha(x)$ be as in Theorem 1. Then,

$$
h \sum_{\gamma=1}^{\left|h^{-1}\right|} f\left(\left\{h^{-1} \alpha(\gamma h)\right\}\right)=\int_{0}^{1} f(x) d x+O\left(h^{1 / 3}\right) \quad \text { as } h \rightarrow 0+.
$$

Proof. Let $h$ with $0<h \leqslant 1 / 8$ be given. With $S_{j, k}$ defined by (2) and $M=$ $\left[h^{-1 / 3}\right]$, the discrepancy $D_{\left[h^{-1}\right]}$ of the points $\left\{h^{-1} \alpha(\gamma h)\right\}, \gamma=1,2, \ldots,\left[h^{-1}\right]$, in $[0,1]$ satisfies

$$
D_{\left|h^{-1}\right|}<<\frac{1}{M}+\sum_{j=1}^{M} \frac{1}{j} S_{j, 0}
$$

according to an inequality in [4], where the implied constant is absolute. It follows from (4) that

$$
D_{\left[h^{-1}\right]}<<\frac{1}{M}+h^{1 / 2} \sum_{j=1}^{M} j^{-1 / 2}<<\frac{1}{M}+h^{1 / 2} M^{1 / 2}<<h^{1 / 3} .
$$

The proof is now completed as in Theorem 1, by using [3, Chapter 2, Theorem 5.1]

Corollary 3. Let $m>0$ be real, and let $\alpha(x)$ be as in Theorem 1. Then,

$$
h \sum_{\gamma=1}^{\left|h^{-1}\right|}\left\{h^{-1} \alpha(\gamma h)\right\}^{m}=\frac{1}{m+1}+O\left(h^{1 / 3}\right) \quad \text { as } h \rightarrow 0+.
$$

In all of the above results, the conditions on $\alpha(x)$ may be relaxed somewhat by assuming only that $\alpha^{\prime \prime}(x)$ exists for all but finitely many points in $[0,1]$, that $0<A \leqslant$ $\left|\alpha^{\prime \prime}(x)\right| \leqslant B$ whenever $\alpha^{\prime \prime}(x)$ exists, and that $\alpha^{\prime \prime}(x)$ changes sign at most finitely often in $[0,1]$. This will only affect the estimation of (3), where one now splits up the sum into a fixed number of subsums in such a way that van der Corput's inequality can be. 
applied to each subsum containing more than one term.

School of Mathematics

The Institute for Advanced Study

Princeton, New Jersey 08540

Department of Mathematics

University of California

Los Angeles, California 90024

Mathematical Sciences Staff

Naval Research Laboratory

Washington, D. C. 20375

1. M. A. FELDSTEIN, Discretization Methods for Retarded Ordinary Differential Equations, Ph.D. Dissertation, University of California, Los Angeles, 1964.

2. E. W. HOBSON, The Theory of Functions of a Real Variable and the Theory of Fourier's Series, Vol. I, 3rd ed., Cambridge Univ. Press, London, 1927.

3. L. KUIPERS \& H. NIEDERREITER, Uniform Distribution of Sequences, Wiley, New York, 1974.

4. H. NIEDERREITER \& W. PHILIPP, "Berry-Esseen bounds and a theorem of Erdös and Turán on uniform distribution mod 1," Duke Math J., v. 40, 1973, pp. 633-649. MR 49 \#2642.

5. J. G. VAN DER CORPUT, "Zahlentheoretische Abschätzungen," Math. Ann., v. 84, 1921 , pp. 53-79. 